\section{Relative Nature of Electromagnetic Radiation}

BY using the method of instantaneous infinitesimal Lorents transformation, we obtain transformation coefficients connecting the two systems $S^{\prime}$ and $S$, with $S^{\prime}$ at rest and $S$ in accelerated motion. Here we give these coefficients for the simplest case, on putting the velocity of $S$ equal to zero after differentiation:

$\frac{\partial x^{\prime}}{\partial x}=1-\frac{x^{\prime} \Gamma}{c^{2}}, \frac{\partial x^{\prime}}{\partial y}=-\frac{y^{\prime} \Gamma}{c^{2}}, \frac{\partial x^{\prime}}{\partial z}=-\frac{z^{\prime} \Gamma}{c^{2}}, \frac{\partial x^{\prime}}{\partial t}=\frac{r^{\prime} \Gamma}{c} ;$

$\frac{\partial y^{\prime}}{\partial x}=0 \quad, \frac{\partial y^{\prime}}{\partial y}=1 \quad, \frac{\partial y^{\prime}}{\partial z}=0 \quad, \frac{\partial y^{\prime}}{\partial t}=0$;

$\frac{\partial z^{\prime}}{\partial x}=0 \quad, \frac{\partial z^{\prime}}{\partial y}=0 \quad, \frac{\partial z^{\prime}}{\partial z}=1 \quad, \frac{\partial z^{\prime}}{\partial t}=0 ;$

$\frac{\partial t^{\prime}}{\partial x}=-\frac{x^{\prime 2} \Gamma}{c^{3} r^{\prime}}, \frac{\partial t^{\prime}}{\partial y}=-\frac{x^{\prime} y^{\prime} \Gamma}{c^{3} r^{\prime}}, \frac{\partial t^{\prime}}{\partial z}=-\frac{x^{\prime} z^{\prime} \Gamma}{c^{3} r^{\prime}}, \frac{\partial t^{\prime}}{\partial t}=1+\frac{x^{\prime} \Gamma}{c^{2}} ;$. where $x^{\prime}, y^{\prime}, z^{\prime}, t^{\prime}$ and $x, y, z, t$ are the co-ordinates of a point relative
to $S^{\prime}$ and $S^{\prime}$ respectively. The values are taken at the time $t^{\prime}-r^{\prime} / c$, and $T$ is the instantaneous acceleration of $S$ along the $x$-direction. We apply these coefficients to the special case of a charged particle placed at the origin of $S$, the accelerated system. The electromagnetic fleld, a six-vector, will be transformed according to

$$
F^{\mu \nu^{\prime}}=\frac{\partial x_{\mu}^{\prime}}{\partial x_{\alpha}} \frac{\partial x_{\mu}^{\prime}}{\partial x_{\beta}} F^{\alpha \beta} .
$$

Let the field of the charge in $S$ be Coulombian, that is,

$$
\vec{E}=\frac{\overrightarrow{e r}}{r^{3}}, \vec{H}=0
$$

Then the field observed by $S^{\prime}$ will be given, by substitution of the coefficients (1) in the equation (2), by

$$
\begin{gathered}
E_{x}^{\prime}=\frac{e x}{r^{\prime 3}}-\frac{e \Gamma}{c^{2} r^{\prime}}+\frac{e \Gamma r^{\prime} x}{c^{2} r^{\prime 2}}, \quad E_{y^{\prime}}=\frac{e y}{r^{\prime 3}} \pm \frac{e \Gamma_{r^{\prime}} y}{c^{2} r^{\prime 2}}, \\
E_{z^{\prime}}=\frac{e z}{r^{\prime 3}}+\frac{e \Gamma r^{\prime} z}{c^{2} r^{\prime 2}} ; \\
H_{x^{\prime}}=0, \quad H_{y^{\prime}}=-\frac{e \Gamma z^{\prime}}{c^{2} r^{\prime 2}}, \quad H_{z}^{\prime}=\frac{e \Gamma y^{\prime}}{c^{2} r^{\prime 2}} ;
\end{gathered}
$$

These are identical with the fleld obtained by solving Maxwell's equations. The fleld of a charge with arbitrary velocity $\vec{v}$ and acceleration $\vec{r}$ is obtained by further applying a finite Lorentz transformation to the above result (3) and is

$$
\begin{aligned}
& \vec{E}^{\prime}=\frac{e\left(1-\beta^{2}\right) o_{1} p}{r^{\prime 3}\left(1-\beta_{\left.r^{\prime}\right)^{3}}\right.}+\frac{e \Gamma_{r^{\prime} o_{1} p}}{c^{2} r^{\prime 2}\left(1-\beta_{\left.r^{\prime}\right)^{3}}\right.}-\frac{e \vec{\Gamma}}{c^{2} r^{\prime}\left(1-\beta_{r^{\prime}}\right)^{2}} ; \\
& \vec{H}^{\prime}=\frac{e\left(1-\beta^{2}\right)\left[\overrightarrow{\left.v x r^{\prime}\right]}\right.}{c r^{\prime 3}\left(1-\beta_{\left.r^{\prime}\right)^{3}}\right.}+\frac{e \Gamma_{r^{\prime}}\left[v x r^{\prime}\right]}{c^{3} r^{\prime 2}\left(1-\beta_{r^{\prime}}\right)^{3}}+
\end{aligned}
$$

$$
\frac{e\left[\vec{\Gamma} x r^{\prime}\right]}{c^{2} r^{\prime 2}\left(\mathrm{I}-\beta_{r^{\prime}}\right)^{2}} ;
$$

where $\overrightarrow{o_{1} p}=\overrightarrow{r^{\prime}}-\vec{v} \frac{r^{\prime}}{c}$, etc.

in complete agreement with the usual classical theory. Therefore the fleld due to an accelerated charge can be obtained from a Coulomb rest fleld by applying the infinitesimal Lorentz transformation (1) and a finite Lorentz transformation.

Next, we wish to find the fleld due to a charge at rest observed by an accelerated observer. To this end, we employ the coefficients $\frac{\partial x}{\partial x^{\prime}}$ etc., which may be obtained directly by solving (1), and are

$$
\begin{array}{lllll}
\frac{\partial x}{\partial x^{\prime}}=1-\frac{x \Gamma}{c^{2}}, \frac{\partial x}{\partial y^{\prime}}=-\frac{y \Gamma}{c^{2}}, \frac{\partial x}{\partial z^{\prime}}=-\frac{z \Gamma}{c^{2}}, \frac{\partial x}{\partial t^{\prime}}=\frac{r \Gamma}{c} ; \\
\frac{\partial y}{\partial x^{\prime}}=0 & , \frac{\partial y}{\partial y^{\prime}}=1 & , \frac{\partial y}{\partial z^{\prime}}=0 & , \frac{\partial y}{\partial t^{\prime}}=0 ; \\
\frac{\partial z}{\partial x^{\prime}}=0 & , \frac{\partial z}{\partial y^{\prime}}=0 & , \frac{\partial z}{\partial z^{\prime}}=1 & , \frac{\partial z}{\partial t^{\prime}}=0 ;
\end{array}
$$$$
\frac{\partial t}{\partial x^{\prime}}=-\frac{x^{2} \Gamma}{c^{3} r}, \frac{\partial t}{\partial y^{\prime}}=-\frac{x y \Gamma}{c^{3} r}, \frac{\partial t}{\partial z^{\prime}}=-\frac{x z \Gamma}{c^{3} r}, \frac{\partial t}{\partial t^{\prime}}=1+\frac{x \Gamma}{c^{2}} ;
$$

Department of Physics

as is easily proved when the two systems instantaneously coincide and with relative velocity equal to zero.

Comparing (1) and (5), we see that the fleld due to a rest charge observed by an accelerated observer is exactly identical with that due to accelerated charge observed by a rest observer, provided that e quantities are relative to the observer in question.

he relative nature of radiation is now obvious and incidentally the relative nature of acceleration is indicated.

We have also investigated the properties satisfying this 'relativity transformation' and shown that the only 'force' existing in Nature is that obeying the central inverse square law and derivations therefrom.

Note added, March 30, by Su-Chin Kiang. The transforma tions discussed above possess group properties with unit acobian. An antisymmetrical tensor of rank 4 can therefore fields are $E^{2}-H^{2}$ and $\vec{E} \cdot \vec{H}$. They represent invariants for systems in arbitrary motion. Taking the simplest case, for example, the field due to an accelerated point charge, they give $\vec{E} \cdot \vec{H}=0$ and $E^{2}-H^{2}$ equal to the rest Coulombian $E^{2}$. HSIN-PEI SOH MU-HSIEN WANG
SU-CHIN KIANG

National University of Chekian

Meitan, Kweichow, China.

$$
\text { March } 15 .
$$

\section{A Non-radiating Motion of a Spinning Electron}

IN both theories referred to in earlier communications ${ }^{1}$, a free spin-particle travels in a suitably chosen frame of reference along a small circle. The point-charge of an electron revolving on a circle the diameter of which is of the order $\frac{\hbar}{m_{e} c}$, where $m_{e}$ is mass of an electron, with the enormous frequency of Schrödinger's Zitterbewegung, would radiate according to the laws of classical electrodynamics with a very high intensity. It is true that a classical model cannot be expected to give a fair account of the radiation emitted by an electron, but such a large radiation without counterpart in Nature would render any correspondence between the classical and the quantummechanical behaviour of an electron impossible. I noted in $1938^{2}$ that the situation changes radically if one takes into account not only the charge of an electron but also its magnetic moment. Under suitable conditions, the radiation due to the revolving magnetic dipole (perpendicular to the plane of the circle) may cancel, approximately at least, the radiation emitted by the revolving point-charge. The argument presented in 1938 was carried through in non-relativistic approximation only, but it can be applied in the general case also,
if we interpret all the quantities involved as referring to the 'restsystem of the circle'.

The argument in brief is as follows. A magnetic dipole while moving produces a transverse electric dipole. The constants of the motion may be adjusted in such a way as to make the superposition of the latter on the point-charge equivalent to the shifting of that charge from the circumference to the centre of the circle. This happens when the angular velocity of the unipole-dipole singularity is $\omega_{\sigma}=$ $\varepsilon / M_{\sigma} c$. Putting $\omega_{\sigma}=M_{\sigma^{c}} / s_{\sigma}$, we get the condition of vanishing radiation in the form

$$
\mu / s=\varepsilon / M_{\sigma} c
$$

$\varepsilon$ being the electric charge of the singularity and $\mu$ the magnitude of its magnetic moment.

In the second $c a s e^{3}, M_{\sigma}$ has to be put equal to the rest-mass of the electron, and thus the radiation vanishes when $\mu / s$ has just the same values as it actually has for an electron. In the first case, however,
the constant mass is not $M_{\sigma}$ but $m_{0}$, which is connected with $M_{\sigma}$ by the relation $m_{0}=M_{\sigma} / \sqrt{1-v_{\sigma^{2}} / c^{2}}$ and (1) becomes $\mu / s=$ $\varepsilon / m_{0} c \sqrt{1-v \sigma^{2} / c^{2}}$. Comparing this with the expression (8) of my earlier communication ${ }^{1}$ we see that in the first model the radiation vanishes only for inflnitesimal 'proper radii of the circle'.

Institute of Theoretical Physics,

Jagellonian University, Cracow

' Weyssenhoff, J. W., Nature, 157, 766 (1946).

2 Weyssenhoff, J. W., Nature, 141, 328 (1938).
3 Weyssenhoff, J. W., Nature, in the press.
All the quantities here are those apparently observed by the accelerated observer, because

$\Gamma\left(S\right.$ relative to $\left.S^{\prime}\right)=-\Gamma\left(S^{\prime}\right.$ relative to $\left.S\right)$
Losses and Wastage in Farm Livestock

IN the past, material for the estimation of the wastage and losses in farm livestock has been collected from the records obtained from survey farms. Another source of valuable information on the question can be obtained from the knackeries which are found in most livestock areas in Great Britain. A West Wales knackery was able to supply a full record of the intake for four years 1942-45 with particulars of the date, source and description into the following (5) steers and heifers). The smaller farm animals - calves, sheep and pigs-were not collected by the knackery as they were considered too small to cover transport costs. This knackery has a collecting area of roughly twenty miles radius. The examination of the records show that a total of 3,116 animals ( 30 per cent table gives the details of the annual intake for each year. 